ISSN 1112-9867

http://www.jfas.info

\title{
DOES EARTHWORMS DENSITY REALLY MODIFY SOIL'S HYDRODYNAMIC PROPERTIES IN IRRIGATED SYSTEMS WITH RECYCLED WATER?
}

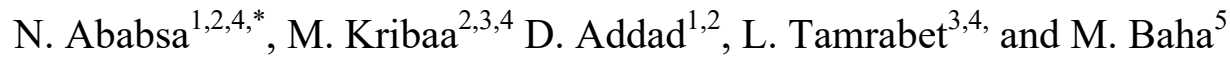 \\ ${ }^{1}$ Université Ferhat Abbas, Setif \\ ${ }^{2}$ Université Abbas Laghrour, Kenchela \\ ${ }^{3}$ Université Larbi Ben Mhidi, Oum El Bouaghi \\ ${ }^{4}$ Laboratoire Ressources Naturelles et Aménagement des Milieux Sensibles \\ ${ }^{5}$ Ecole National Superieur De Kouba,
}

Received: 12 February 2016 / Accepted: 22 April 2016 / Published online: 01 May 2016

\begin{abstract}
Our study has the general objective to understand the impact of the valuation of treated water on earthworm abundance and total porosity of the soil and the effect of the interaction between these two physical-biological components of the hydrological functioning of soils. It was carried out on the meadows soils of the valley of Wadi Bousselam.

Although the treated water has high organic and particulate filler, it improved the earthworm abundance, total porosity and hydraulic conductivity of the soil.
\end{abstract}

Keywords: meadows soils; earthworm density; soil morphology; treated water; hydrodynamic soil characteristics.

Author Correspondence, e-mail: welnim@yahoo.fr

doi: http://dx.doi.org/10.4314/jfas.v8i2.29 


\section{INTRODUCTION}

Dans les hautes plaines Sétifiennes, les prairies naturelles se localisent sur l'étage bioclimatique compris entre $200 \mathrm{~mm} / \mathrm{an}$ et $600 \mathrm{~mm} / \mathrm{an}$ au nord et se repèrent dans les bords d'oueds et les bas-fonds. Leurs superficies ont connu une forte régression depuis l'indépendance où elles occupent une superficie de 24820 ha ce qui représente $1.07 \%$ de la SAT de la wilaya de Sétif.

L'irrigation des prairies est une activité très importante et même primordiale, vu qu'elles se trouvent dans une zone semi-aride, caractérisée par de fortes amplitudes thermiques, des pluies irrégulières ce qui impliquait de longues périodes de sécheresse. Depuis deux décennies la valorisation des eaux traitées pour l'irrigation de ces prairies connait un grand développement. C'est une solution alternative et respectueuse des ressources en eau et de l'environnement car elle apporte l'eau et les éléments fertilisants en même temps une sorte de fertigation ou ferti-irrigation.

Le sol est un écosystème naturel qui évolue sans cesse spatialement et temporellement, et c'est l'étude de l'interaction entre ces composantes physiques chimiques et biologiques qui aide à comprendre le fonctionnement de cet écosystème fragile et non renouvelable.

Les vers de terre sont une composante majeure des communautés de la faune du sol dans la plupart des écosystèmes et comprennent une grande proportion de la biomasse des macrofaunes. [1-5].

Les vers de terre sont donc des acteurs importants dans les sols, où ils assurent de nombreuses fonctions écosystemiques. Ils affectent la structure et la fertilité des sols [5]. Par le biais de diverses activités les vers de terre peuvent influencer la morphologie ainsi que les propriétés du sol (physique chimique et biologique) bien que l'ampleur de cette influence peut varier entre les types de sol [6].

Da Silva, [7] indique qu'il est important de connaitre et d'estimer leur diversité et leur comportement dans les sols, en particulier dans un contexte où les sols sont de plus en plus contaminés et influencés par les activités anthropiques.

L'objectif de cette étude consiste à évaluer l'impact de la valorisation des eaux traitées issue d'une station d'épuration à boue activée sur i) l'abondance lombricienne, ii) la porosité des 
sols considérée comme l'un des aspects essentiels de la morphologie des sols [8], iii) ainsi que l'interaction de ces deux composantes sur les caractéristiques hydrodynamiques qui se rapportent à la rétention et au transfert d'eau.

\section{MATERIELS ET METHODES}

\subsection{Milieu d'étude}

Notre recherche a été menée dans les prairies pré urbaines de la ville de Sétif localisées dans les hautes plaines céréalières de l'est Algérien à $900 \mathrm{~m}$ d'altitude, caractérisée par de fortes amplitudes thermiques, des pluies irrégulières ce qui impliquait de longues périodes de sécheresse.

La station d'épuration des eaux usées crée en 1996, traite plus de la moitié des effluents générés par la ville et déverse les eaux traitées dans oued Boussellem. Donc la partie aval de ce cours d'eau est alimentée par les eaux traitées depuis vingt ans et parce qu'il présente une source permanente et gratuite d'eau et de fertilisants, cet Oued est utilisé pour l'irrigation de l'espace prairial ce qui rend, d'après Boudjenouia et al. [9], les prairies naturelles plus fréquentes dans la région.

Pour notre étude nous avons choisi deux sites le premier en aval de la station d'épuration où les sols sont irrigués par les eaux traitées rejetées dans l'oued (ET), le seconde dans la partie aval d'oued Boussellam où les sols des prairies reçoivent des eaux qui ont comme origine les eaux pluviales utilisés comme témoin pour confirmer l'effet des eaux traitées sur les caractéristiques des sols étudiées. Chaque site fait l'objet de plusieurs répétitions pour l'ensemble des paramètres.

\subsection{Méthodologie}

Au niveau des deux sites une caractérisation physico-chimique des eaux a été effectuée sur des échantillons prélevés dans des bouteilles en polyéthylène de 1,5 litre.

La caractérisation globale des communautés lombriciennes, a été effectuée par des échantillonnages sur chaque site suivant la méthode du formol couplée à un tri manuel. Elle est appréciée en termes de densité moyenne, catégories écologiques, et âge ratio exprimée en nombre d'individus par $\mathrm{m}^{2}$. Les vers ont ensuite été identifiés. 
Les courbes de rétention d'eau ont été déterminées sur des échantillons de sol à différents potentiels de succions, $\theta(0.3), \theta(0.78), \theta(1), \theta(3.2), \theta(31.6)$ et $\theta(100)$ à l'aide de l'appareil de la presse à membrane.

La mesure de la conductivité hydraulique du domaine proche de la saturation est réalisée in situ à l'aide d'un infiltromètre mono disque d'un diamètre moyen de $80 \mathrm{~mm}$ en contrôlant la pression à la surface du sol. Les mesures d'infiltration sont faites à 4 potentiels de différentes pressions $0.06,0.3,0.6$ et $1.5 \mathrm{kPa}$

La porosité a été calculée en se basant sur les mesures de la densité apparente et à une densité réelle de $2,65 \mathrm{~g} / \mathrm{cm}^{3}$.

\section{RÉSULTATS ET DISCUSSION}

\subsection{Qualité des eaux}

Dans cette étude notre objectif est de quantifier les effets de la valorisation des eaux traitées sur les propriétés hydrobiophysique des sols pour cette raison nous nous sommes intéressé à la charge organique et particulaire des eaux d'irrigations, ainsi que le $\mathrm{pH}$ et la conductivité électrique $(\mathrm{CE})$ des eaux ont été mesurés.

Les caractéristiques des eaux sont regroupées dans la figure (1). Cette dernière révèle que le $\mathrm{pH}$ des eaux est alcalin, mais il ne dépasse guère la norme Algérienne des eaux de surface.

La comparaison des valeurs de la conductivité électrique (CE) enregistrées avec la norme Algérienne des eaux superficielles montre qu'elles sont inférieures à la norme considérée comme valeur limite des eaux superficielles $(2800 \mu \mathrm{S} / \mathrm{cm})$. Avec une valeur moyenne plus élevée enregistrée pour le site des eaux de surface.

Une charge organique, qui se rapporte à la $\mathrm{DBO} 5$ et DCO, très élevée a été enregistrée pour le site des eaux traitées. Elle dépasse largement les deux normes Algérienne des eaux de surface et des eaux traitées destinées à l'irrigation. Alors que des valeurs faibles sont marquées par le site des eaux de surface.

Pour les matières en suspension les deux types d'eau sont très chargés en matières particulaire avec des concentrations plus élevées pour les eaux traitées. 

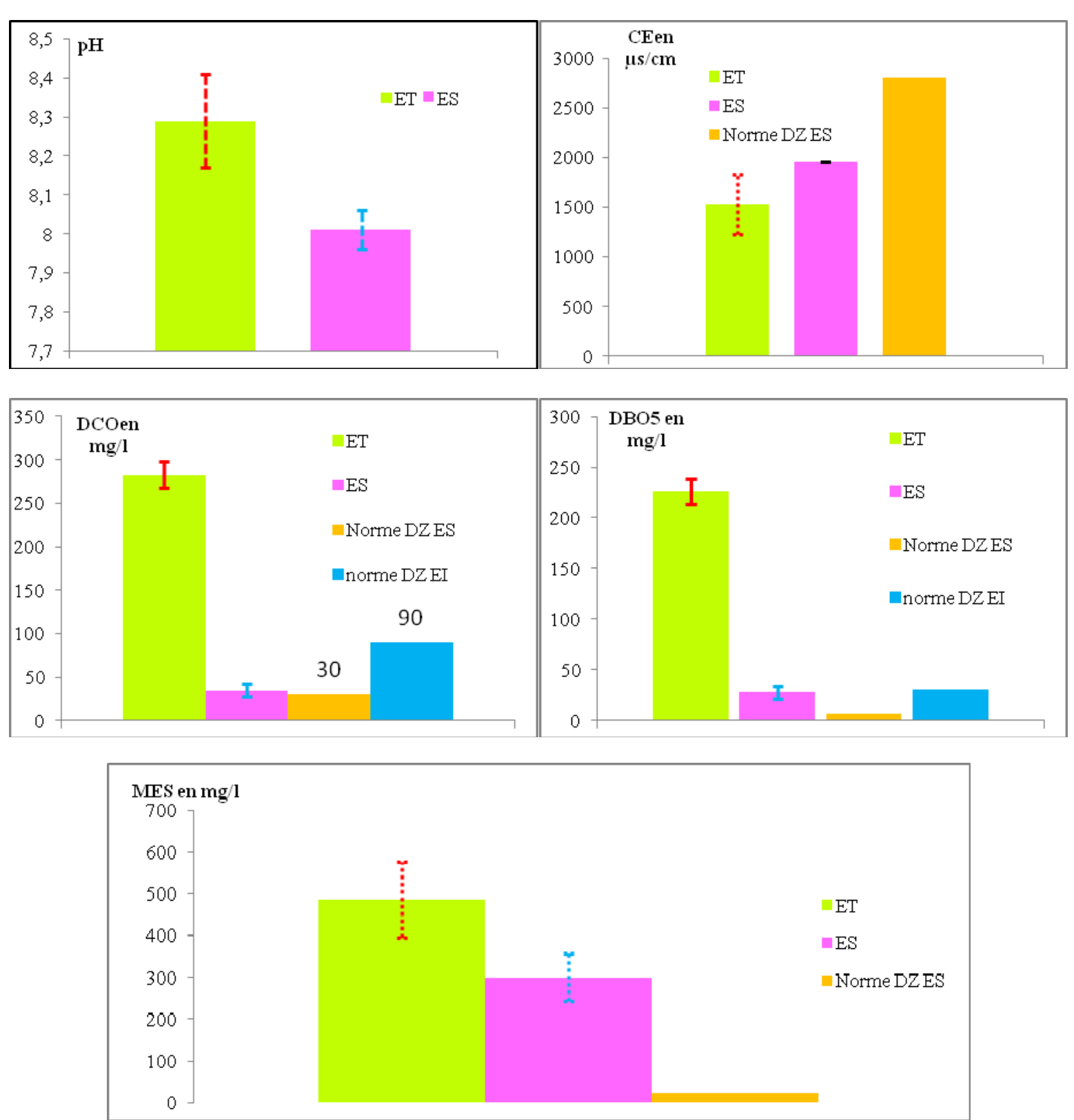

Fig.1. Caractéristiques des eaux d'irrigation (ET : eau traitée, ES : eau de surface, norme algérienne des eaux de surface)

\subsection{Populations lombriciennes}

Au total 138 vers ont été collectés dans les deux sites étudiés, avec des valeurs moyennes de 26 et 20 vers $/ \mathrm{m}^{2}$ dans les deux sites irrigués à l'eau traitée et à l'eau de surface respectivement, dont la distribution par sites est résumée dans la figure (2), cette dernière montre clairement que la densité des vers est plus élevée pour le site irrigué avec les effluents traités. 
Globalement trois espèces de vers de terre ont été identifiées dont deux endogées et une espèce anécique, la figure (3) démontre bien la dominance de la catégorie écologique des endogées.

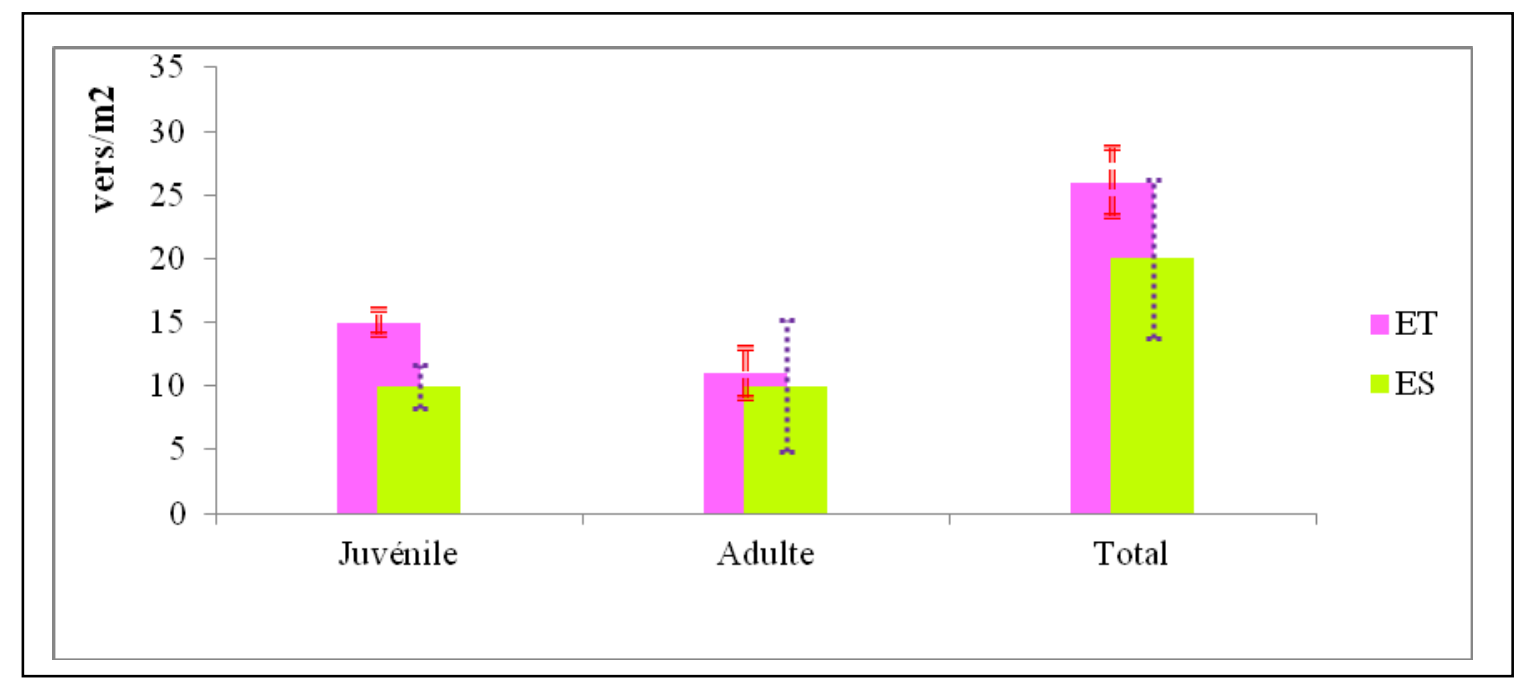

Fig. 2. Abondance lombricienne avec proportion de formes juvéniles et adultes dans les deux sites (ET : sols irrigué à l'eau traitée, ES : sols irrigué à l'eau de surface)

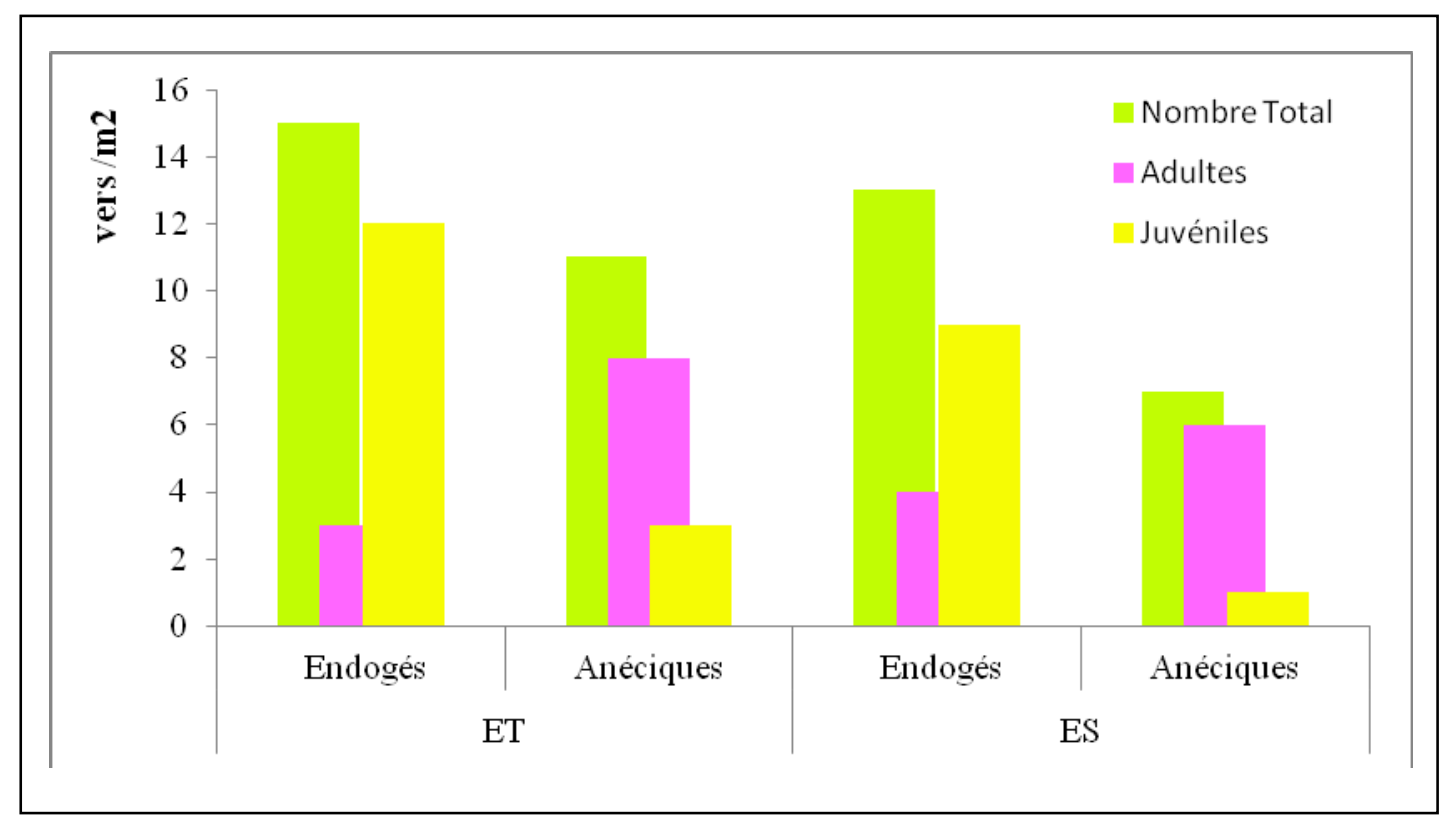

Fig.3. Catégories écologiques des vers échantillonnés dans les deux sites (ET : sols irrigué à l'eau traitée, ES : sols irrigué à l'eau de surface) 


\subsection{Porosité totale des sols estimée à partir des mesures de densité}

La porosité du sol joue un rôle important dans les échanges hydriques et gazeux, ainsi que dans le développement racinaire. Les valeurs moyennes de la porosité telles que présentées dans la figure (4) montrent une légère différence entre les deux traitements.

La forte porosité mesurée dans les sols irrigués à l'eau traitée est liée à la présence d'une activité lombricienne importante assurant une certaine régénération de la structure, ce qui correspond à une augmentation de la bioturbation.

Nos résultats confirment que la porosité est influencée par l'abondance lombricienne. Lorsque les communautes lombriciennes (endogés) sont plus abondantes elles plus de porosité. Ces résultats suivent ceux de Bottinelli et al. [10] et Peres et al. [11] qui ont rapporté que les vers de terre participent à l'amélioration de la porosité des sols.

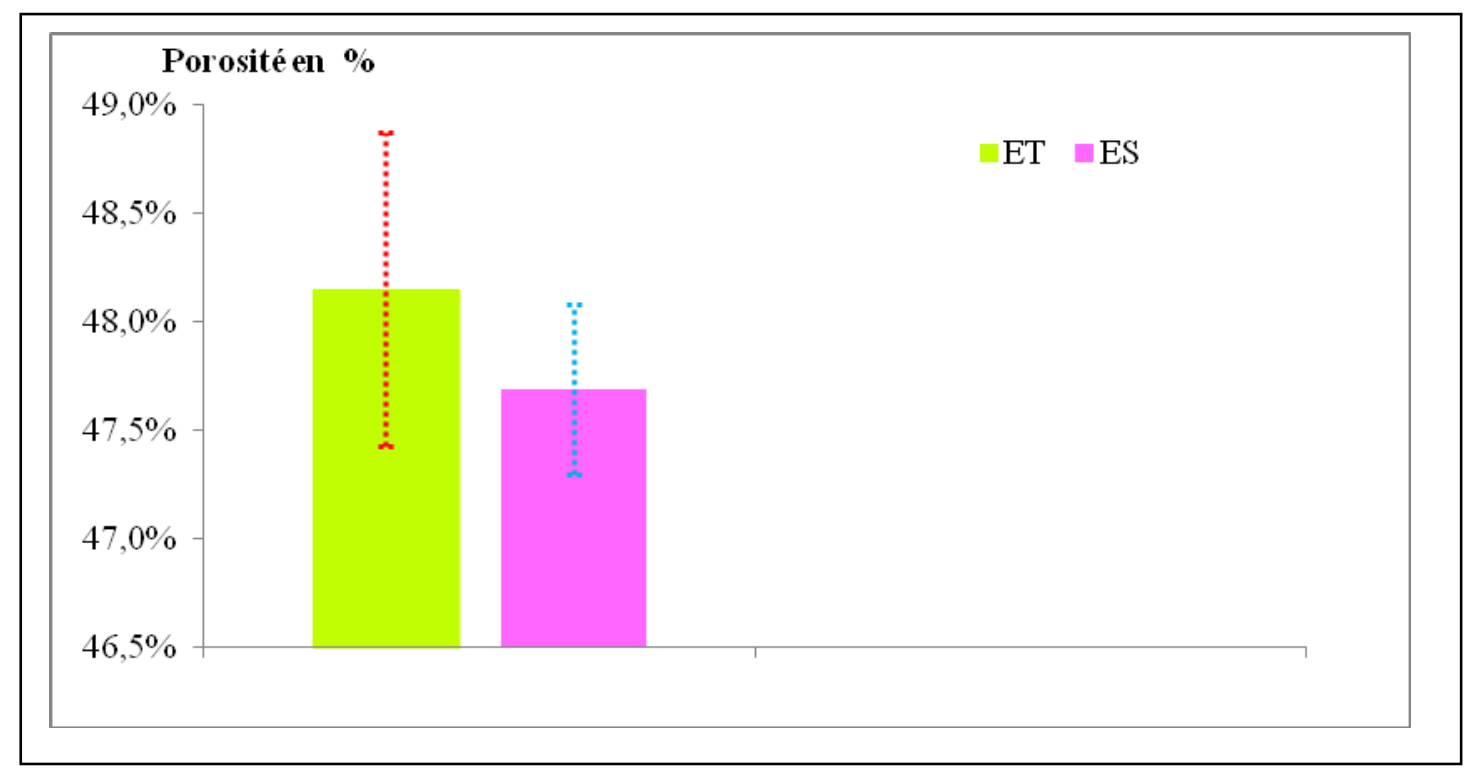

Fig.4. Porosité totale des sols irrigués avec les deux types d'eau (ET : sols irrigué à l'eau traitée, ES : sols irrigué à l'eau de surface)

\subsection{Caractéristiques hydriques}

Le comportement hydrodynamique d'un sol est généralement décrit par les deux paramètres : la conductivité hydraulique et la courbe de rétention d'eau.

\subsubsection{Conductivité hydraulique au voisinage de la saturation}

La conductivité hydraulique proche à la saturation est choisie pour apprécier la perméabilité des sols irrigués avec les deux types d'eau, afin de déterminer l'impact du type d'eau, de la 
structure (porosité) et des peuplements lombricienne sur le fonctionnement hydrique des sols étudiés.

Les deux succions $(-0.06,-0.3 \mathrm{kPa})$ mettent en évidence une nette supériorité pour les sols irrigués à l'eau traitée et une légère supériorité pour la succion $(-0.6 \mathrm{kPa})$. Alors que pour la succion $(-1.5 \mathrm{kPa})$ une légère supériorité est observée pour les sols irrigués à l'eau de surface (figure 5). On en déduit que c'est au niveau des deux succions (-0.06,- $0.3 \mathrm{kPa})$ qu'apparaissent l'effet des vers de terre et du type d'eau d'irrigation.

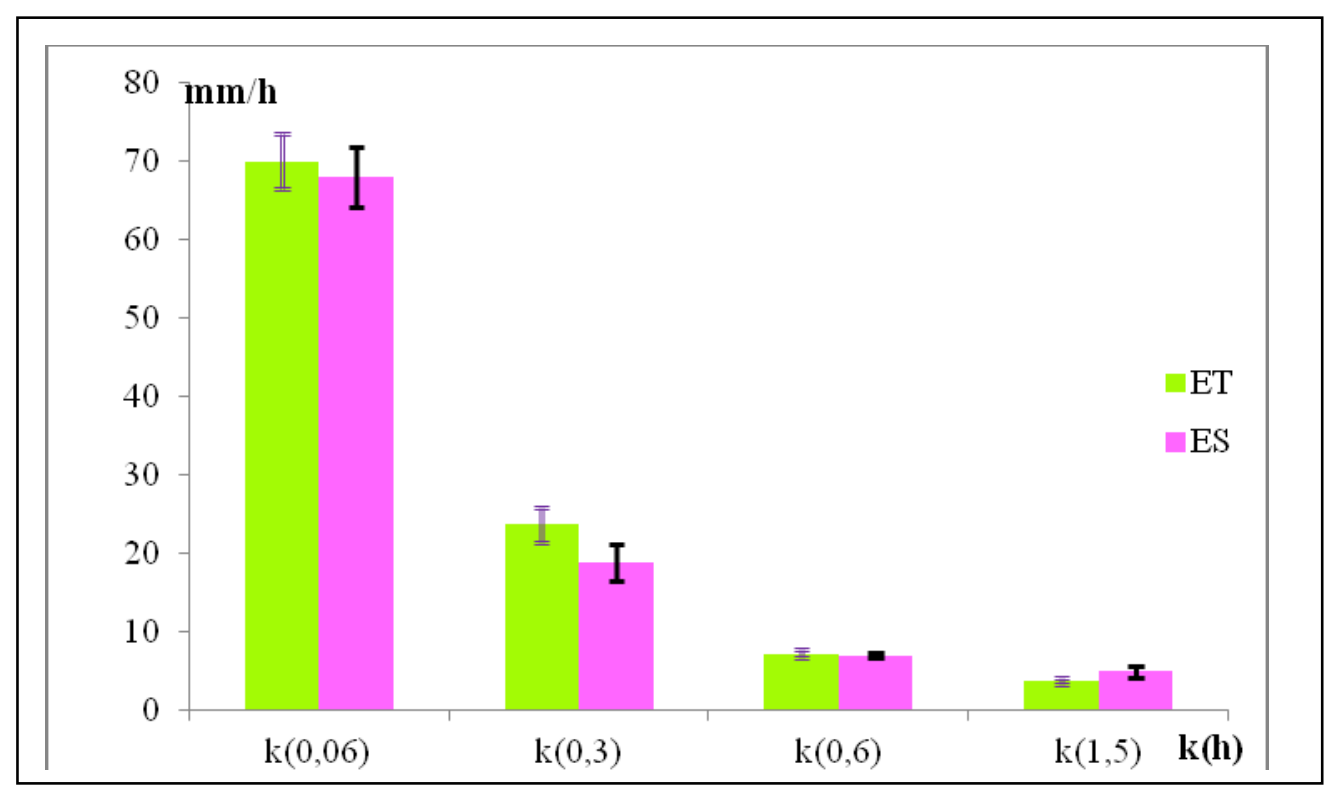

Fig.5. Valeurs moyennes de la conductivité hydraulique au voisinage de la saturation (ET : sols irrigué à l'eau traitée, ES : sols irrigué à l'eau de surface)

Généralement, La capacité d'infiltration rapide d'un sol est estimée plutôt par la macroporosité. Certains auteurs [12] et [13], ont signalé que les galeries creusés par l'activité de bioturbation des lombrics participaient aux transferts hydriques.

\subsubsection{Courbe de rétention en eau}

Les courbes caractéristiques de l'humidité volumétrique des sols des prairies irriguée avec les deux types d'eau sont présentées dans la figure (6). Pour les succions $\theta(0,3), \theta(0.78)$, $\theta(1)$ et $\theta(3,2)$ les sols irrigués avec les eaux de surface retiennent plus d'eau que ceux irrigués à l'eau traitée. Alors que pour les deux succions $\theta(31,6)$ et $\theta(100)$ aucune différence n'est enregistrée entre les deux sites. Donc en termes de rétention d'eau, l'eau traitée n'a aucune 
influence. Jangorzo et al. [14] ont indiqué que la grande proportion des pores capillaires et leur connectivité induisent une meilleure rétention d'eau.

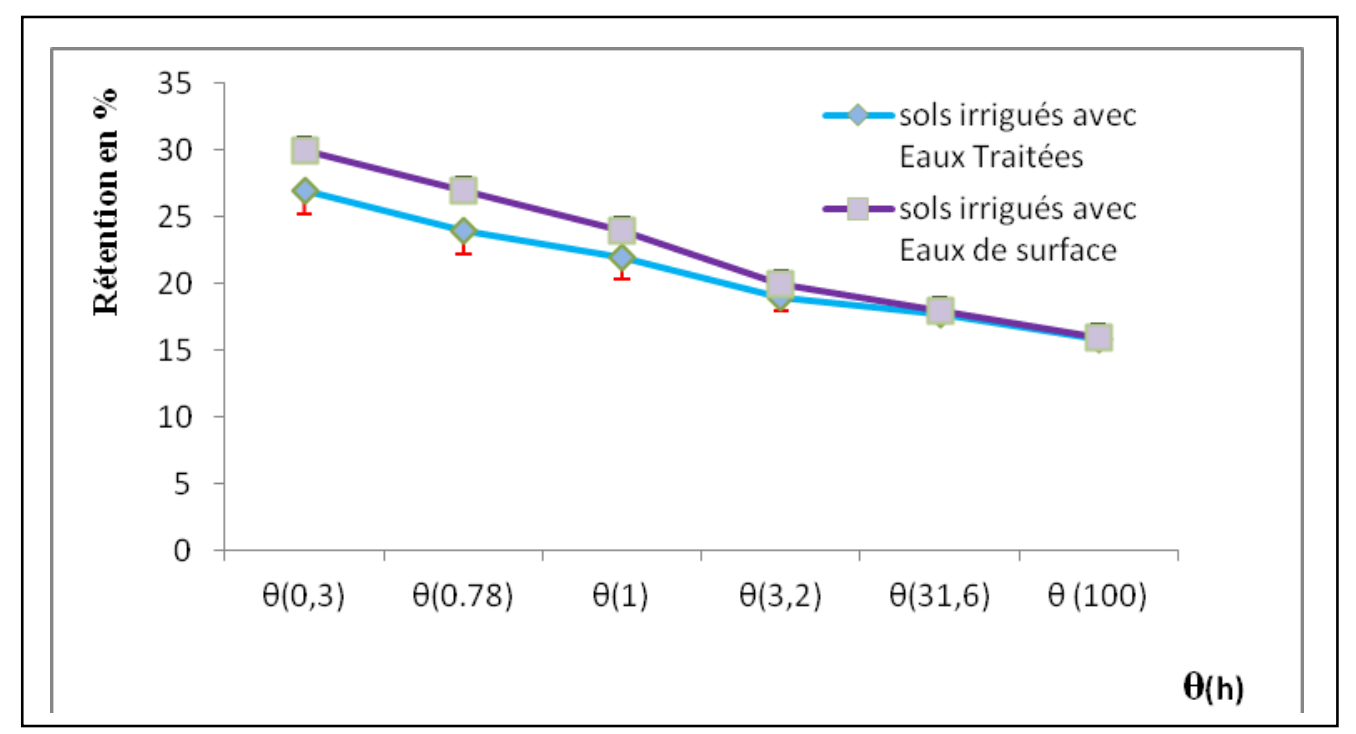

Fig.6. Courbes de rétention d'eau pour les sols irrigués avec les deux types d'eau (ET : sols irrigué à l'eau traitée, ES : sols irrigué à l'eau de surface)

\subsection{Interaction entre la densité lombricienne et la porosité totale du sol et l'influence sur le fonctionnement hydrique des sols}

Nos résultats mettent en évidence que l'utilisation des eaux traitées pour l'irrigation des sols des prairies influence l'augmentation de la densité des lombrics cette dernière s'accompagne d'une sensible augmentation de la porosité totale des sols ainsi une amélioration du fonctionnement hydrique des sols qui se rapporte seulement à la conductivité hydraulique, car aucune amélioration de la rétention en eau n'est enregistrée avec l'utilisation des eaux traitées. Pères, [15] a indiqué l'absence de corrélation significative entre la population lombricienne et la conductivité hydraulique.

Donc on en déduit que la charge organique et particulaire très élevée des eaux traitées n'a pas des répercussions négatives sur la porosité totale et la conductivité hydraulique. Ce qui est contradictoire avec les travaux de certains auteurs [16], [17], [18], [19]. Tandis que Minhas et Samra, [20] ont indiqué des résultats qui concorde avec nos résultats.

Cela peut être justifié par l'abondance des communautés lombricienne qui ont participés à la dégradation de cette charge organique et particulaire. Plusieurs auteurs indiquent que les 
activités des vers de terre améliorent la structure du sol et augmentent l'infiltration [21], [22]. Cela concorde à notre travail.

\section{CONCLUSION}

Notre étude a visé à apprécier l'impact de la valorisation des eaux traitées sur l'abondance lombricienne et la porosité totale des sols et l'effet de l'interaction entre ces deux composantes physico-biologiques sur le fonctionnement hydrique des sols.

Elle a permis de quantifier le rôle important des eaux traitées, malgré la charge organique et particulaire très élevée, dans l'amélioration de la densité lombricienne, cette communauté lombricienne via l'activité de bioturbation a entrainé des changements de la porosité totale ce qui avait un impact positif sur les caractéristiques hydrodynamiques rapportées à la conductivité hydraulique dans les conditions proches à la saturation. Alors que les eaux traitées n'induisent aucune amélioration de la rétention d'eau.

\section{REFERENCES}

[1] Edwards, C. A. et Bohlen, P. J., Biology and Ecology of Earthworms 3rd ed. Chapman and Hall, London1996, 426 p.

[2] Lavelle P., Spain, A.V., Soil Ecology. Kluwer Academic Publishers, the Netherlands, 2001.

[3] Edwards, C.A., Earthworm Ecology (2nd Edition). C.R.C. Press, Boca Raton, Fl., London, New York, Washington,2004. 448 p.

[4] Smith, R. G., McSwiney, C. P., Grandy, A. S., Suwanwaree, P., Snider, R. M and Robertson, G. P., Diversity and abundance of earthworms across an agricultural land-use intensity gradient. Soil and Tillage Research, 100, 2008, pp 83-88.

[5] Bhadauria T. and Saxena K. G. Role of Earthworms in Soil Fertility Maintenance through the Production of Biogenic Structures. Applied and Environmental Soil Science, 2010, 7 p, doi:10.1155/2010/816073.

[6] Oades J.M., The role of biology in formation, stabilization and degradation of soil structure. Geoderma 56, 1993, pp 377-400.

[7] Da Silva E., Interactions « sol - vers de terre » et dynamique du mercure en Guyane 
française, 2013. Thèse doc. Univ. Paris Est. 273 p.

[8] Ruellan A., Morphologie et fonctionnement des sols: quelques réflexions pour l'avenir de la Pédologie. Cah. O.R.S.T.O.M., sér. Pédol., vol. XX, no 4, 1983, pp266-270.

[9] Boudjenouia A. Fleury A. Tacherift A. Le statut de l'espace agricole périurbain à Sétif (Algérie) : réserve foncière ou projet urbain. Cahiers Agricultures vol. 15, n², 2006, pp 221- 226.

[10] Bottinelli, N., Henry-des-Tureaux, T., Hallaire, V., Mathieu, J., Benard, Y., Duc Tran, T., Jouquet, P., Earthworms accelerate soil porosity turnover under watering conditions. Geoderma 156 (1-2), 2010, pp 43-47.

[11] Peres, G., Cluzeau, D., Curmi, P., Hallaire, V., 1998. Earthworm Activity and Soil Structure Changes due to Organic Enrichments in Vineyard Systems. Biol. Fertil. Soils, 27,pp 417-424.

[12] Francis, G.S. and Fraser, P.M., 1998. The effects of three earthworm species on soil macroporosity and hydraulic conductivity. Appl. Soil Ecol., 10(1-2): 11-19.

[13] Capowiez, Y., Cadoux, S., Bouchant, P., Ruy, S., Roger-Estrade, J., Richard, G. and Boizard, H., 2009. The effect of tillage type and cropping system on earthworm communities, macroporosity and water infiltration. Soil Tillage Res., 105(2): 209-216.

[14] Jangorzo NS., Watteau F., Schwartz CH. 2013. Evolution of the pore structure of constructed technosols during early pedogenesis quantified by image analysis. Geoderma 207-208, 180-192.

[15] Pères G., identification et quantification in situ des interactions entre la diversité lombricienne et la macro-bioporosité dans le context polyculture Breton.influence sur le fonctionnement hydrique du sol.these Doc.Univ. Rennes1, 2003, 200 p.

[16] Wang Z., Chang A.C., Wu L., Crowley D. Assessing the Soil Quality of Long-Term Reclaimed Wastewater-Irrigation Cropland, Geoderma, Vol. 114, 2003, pp. 261-278.

[17] Viviani G. \& Iovino M. Wastewater Reuse Effects on Soil Hydraulic Conductivity, Journal of Irrigation and Drainage Engineering, Vol. 130, 2004, pp. 476-482.

[18] Sou Y.M. Recyclage des eaux usées en irrigation : potentiel fertilisant,_risques sanitaires et impacts sur la qualité des sols. Thèse DOC. É PF. Lausanne, 2009, 162 p. 
[19] Molahoseini H., 2014. Long-Term Effects of Municipal Wastewater Irrigation on Some Properties of a Semiarid Region Soil of Iran. International Journal of Scientific Engineering and Technology (ISSN : 2277-1581) Volume No.3 Issue Nº, pp 444 - 449

[20] Minhas P.S. \& Samra, J.S. Wastewater use in peri-urban agriculture: impacts and opportunities. Tech. Bull. No. 2. Central Soil salinity Research Institute, Karnal 132001, India, 2004, 65 p.

[21] Syers J.K., Springett J.A. Earthworm ecology in grassland soils. In: Satchell, J.E. (Ed.), Earthworm Ecology: From Darwin to Vermiculture. Chapman \& Hall, London, 1983, pp. 67-83.

[22] Edwards W.M., Shipitalo M.J. Consequences of earthworms in agricultural soils: aggregation and porosity. In: Edwards, C.A. (Ed.), Earthworm Ecology. CRC Press, Boca Raton, FL., 1998, pp. 147-161.

\section{How to cite this article:}

Ababsa N, Kribaa M, Addad D, Tamrabet L. Baha M. Does earthworms density really modify soil's hydrodynamic properties in irrigated systems with recycled water?. J. Fundam. Appl. Sci., 2016, 8(2), 627-638. 